\title{
Energy-Efficient Traffic Grooming in Bandwidth Constrained IP over WDM Networks
}

\author{
Bin Chen ${ }^{1}$, Zijian Yang ${ }^{1}$, Rongping Lin ${ }^{2}$, Mingjun Dai ${ }^{1}$, Xiaohui Lin ${ }^{1}$, Gongchao Su ${ }^{1}$ \\ and Hui Wang ${ }^{1}$ \\ ${ }^{1}$ Engineering Center for Ubiquitous Computing and Intellighent Networking, College of Information Engineering, \\ Shenzhen University, CHINA \\ [e-mail: bchen@szu.edu.cn, xhlin@szu.edu.cn] \\ ${ }^{2}$ School of Communication and Information Engineering, University of Electronic Science and Technology of \\ China, CHINA \\ *Corresponding author: Xiaohui Lin
}

Received September 23, 2017; revised January 31, 2018; accepted February 5, 2018; published June 30, 2018

\begin{abstract}
Minimizing power consumption in bandwidth limited optical traffic grooming networks is presented as a two-objective optimization problem. Since the main objective is to route a connection, the network throughput is maximized first, and then the minimum power consumption solution is found for this maximized throughput. Both transparent IP over WDM (Tp-IPoWDM) and translucent IP over WDM (Tl-IPoWDM) network may be applied to examine such bi-objective algorithms. Simulations show that the bi-objective algorithms are more energy-efficient than the single objective algorithms where only the throughput is optimized. For a Tp-IPoWDM network, both link based ILP (LB-ILP) and path based ILP (PB-ILP) methods are formulated and solved. Simulation results show that PB-ILP can save more power than LB-ILP because PB-ILP has more path selections when lightpath lengths are limited. For a Tl-IPoWDM network, only PB-ILP is formulated and we show that the Tl-IPoWDM network consumes less energy than the Tp-IPoWDM network, especially under a sparse network topology. For both kinds of networks, it is shown that network energy efficiency can be improved by over-provisioning wavelengths, which gives the network more path choices.
\end{abstract}

Keywords: IP over WDM network, Traffic grooming, Bi-objective, Power consumption

The research was jointly supported by research grant from Natural Science Foundation of China (61575126, 61401070), Natural Science Foundation of Guangdong Province (2015A030313552), the Key Project of Department of Education of Guangdong Province (2015KTSCX121), Foundation of Shenzhen City (201708183000087). 


\section{Introduction}

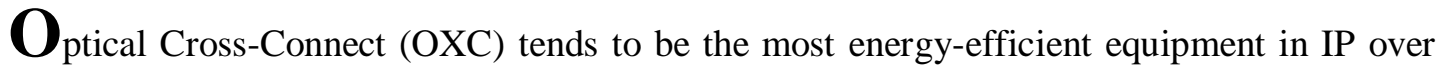
WDM networks. A Micro-Electro-Mechanical System (MEMS) based OXC consumes just 10 $\mathrm{pJ}$ or less for switching one bit, whereas a typical high-end core router would consume around $20 \mathrm{~nJ}$ [1]. As the transmission speed of a wavelength channel increases, the relative energy efficiency of an OXC will be even more significant. Switching traffic by OXCs rather than by routers would therefore be the key to improve the energy efficiency for an IP over Wavelength-Division Multiplexing (WDM) network [2]. Note that increasing the energy efficiency of the network will be important for "Green" traffic grooming [1].

If we are to switch traffic using OXCs rather than routers in IP over WDM networks, then the small granularity traffic of the IP layer needs to be groomed into large volume traffic for efficient and practical optical switching. For an IP over WDM network, traffic grooming has been proposed earlier for network cost saving [3-4], which would then effectively maximize the network throughput or minimize the network blocking ratio in a given IP over WDM network. With the current growth in the volume of Internet traffic, reducing the power consumption for this is expected to become important for planning and deployment of IP over WDM networks [5]. In [6], the authors have shown that merely minimizing the number of lightpaths used or the amount of traffic switched is not efficient in terms of overall power consumption. A power aware grooming strategy is necessary for optimizing the network's overall power consumption. In [2], efficient approaches such as one using mixed integer linear programming (MILP) models and heuristics are developed based on a lightpath bypass concept. In [7], the authors studied the network energy savings if some customers switch to energy saving services. In [8], four optical core network architectures were compared by giving their respective integer linear programming (ILP) formulations. In [9], the energy costs of IP over mixed-line-rate (MLR) WDM networks were investigated. Green traffic grooming is also studied in [10] for elastic optical network with both an ILP-based problem formulation and various heuristic grooming policies. Since the real traffic load fluctuates periodically, another way to save energy is to put idle equipment into a sleep mode of operation [11-13]. In this situation, traffic grooming would still be important for improving the optical channel utilization.

It may be noted that, especially in the static provisioning scenario, most of the earlier studies on energy-efficient traffic grooming either do not consider the blocking situation, or assume that all connection requests can be admitted [2][6-11]. In the dynamic provisioning scenario, the authors try to study the trade-off between energy saving and network blocking performance [14] in transparent WDM networks. Simulation results show that saving energy actually increases network blocking. This side effect has also been shown in [15-18] for IP over WDM networks. It may be noted that from the point of view of network management and operation, blocking a connection request may not be acceptable if the corresponding network bandwidth is still available.

In this work, we use an ILP based approach to study energy-efficient static traffic grooming in a bandwidth limited IP over WDM network, so that the network's power consumption is minimized without sacrificing its throughput. Based on the lexicographic approach [19-20], a two-phase ILP method is adopted for minimizing power consumption in a bandwidth limited IP over WDM network. As the throughput objective has higher priority than the power consumption objective, the network throughput is maximized first. 
Subsequently, the network's power consumption is minimized, but only to the extent that the maximum value of the network throughput is remained.

Under the transparent IP over WDM (Tp-IPoWDM) network, we tried two kinds of ILP formulations, i.e. the PB-ILP (path based ILP) formulation and the LB-ILP (link based ILP) formulation. Although it is well known that PB-ILP may only provide a sub-optimal solution when only a limited number of paths are considered [21], we were surprised to find that the PB-ILP has better performance in dense networks. (The justification for this is given later in the paper.) For translucent IP over WDM (Tl-IPoWDM) networks, it is shown that the network can manipulate 3R (re-timing, re-amplification and re-shaping) regenerators to improve its energy efficiency.

This paper is an extension of our previous work [20], in which we had for the first time, introduced the lexicographic multi-objective ILP for solving the power consumption minimization problem in bandwidth limited IP over WDM networks. In the present paper, a comparison between LB-ILP and PB-ILP in Tp-IPoWDM networks is included and the formulation is extended to consider four layer Tl-IPoWDM network. Our contributions in this paper can be summarized as follows:

1) To the best of our knowledge, this is the first bi-objective algorithm to further decrease the power consumption of the IP over WDM network after its throughput is maximized.

2) We are the first to show that the Tp-IPoWDM network under PB-ILP can consume less energy than that under LB-ILP when the length of a transparent lightpath is limited. This is because PB-ILP has more path choices than LB-ILP under the transparent lightpath length limit in WDM networks.

3) According to all the simulation results, we found that increasing path selection is important for saving energy in IP over WDM networks. In the dense network topology, the Tl-IPoWDM network can save a significant amount of power than the Tp-IPoWDM network when the traffic load is close to the network capacity. However, if the network capacity or the number of wavelengths per fiber continues to increase further, the saving energy effect declines. In the sparse network topology, the power consumption of the Tl-IPoWDM network is lower than that of the Tp-IPoWDM network, even when there is more than enough network capacity. That is because to increase link capacity or the number of wavelengths can not increase transparent lightpath choices under the sparse network topology. The $3 \mathrm{R}$ regenerators are important for saving energy in sparse Tl-IPoWDM networks.

This paper is organized as follows. The network power consumption model used in this work is described in Section II. In Sec. III, the PB-ILP formulations and LB-ILP formulations are introduced for the Tp-IPoWDM network. In Sec. IV, simulation results of the proposed methods are discussed. In Sec. V, the PB-ILP formulations under the Tl-IPoWDM network are introduced. In Sec. VI, the network performances are compared between Tl-IPoWDM and Tp-IPoWDM networks. Sec. VII concludes the paper.

\section{The Power Consumption Models of IP over WDM Networks}

This work studies Tp-IPoWDM and Tl-IPoWDM networks [8] as shown in Fig. 1 (a) and Fig. 2(a). In each node, an OXC is connected to an IP core router via transponders which perform optical-electrical-optical conversion. 
In the Tp-IPoWDM network as shown in Fig. 1(b), 3 layers are considered, i.e. the fiber layer, the transparent lightpath (TPL) layer and the IP layer. In the fiber layer, OXC nodes are connected by fibers for constructing the network's physical topology. In the TPL layer, a virtual link is composed of a transparent lightpath, which may be switched from incoming fibers to outgoing fibers (as long as the wavelength continuity constraint is satisfied), or may be terminated in the connected routers. The end nodes of a virtual link in the TPL layer are the transponders. The transmission length of the virtual link is limited by the signal impairments of the optical transmission system. A transparent lightpath must be terminated by a transponder before its signal quality falls below the acceptable level [22]. In the IP layer, an IP connection is established between IP nodes (core routers) based on the virtual topology provided by the TPL layer. Core routers switch an IP connection between transparent lightpaths until it reaches the destination.

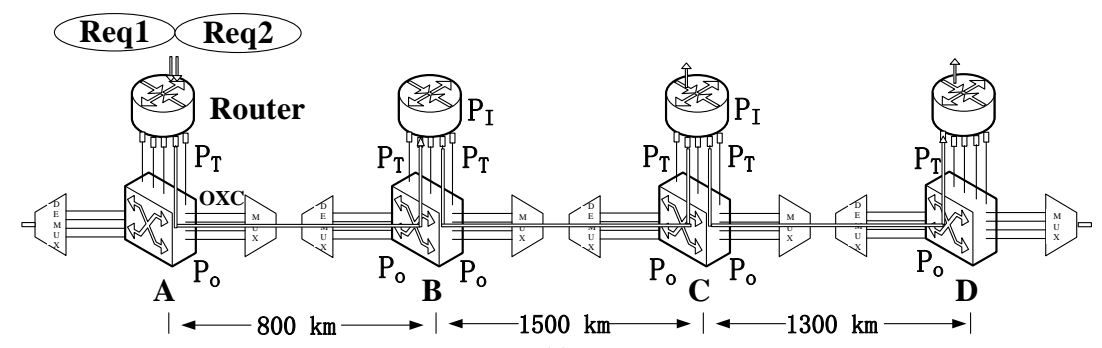

(a)

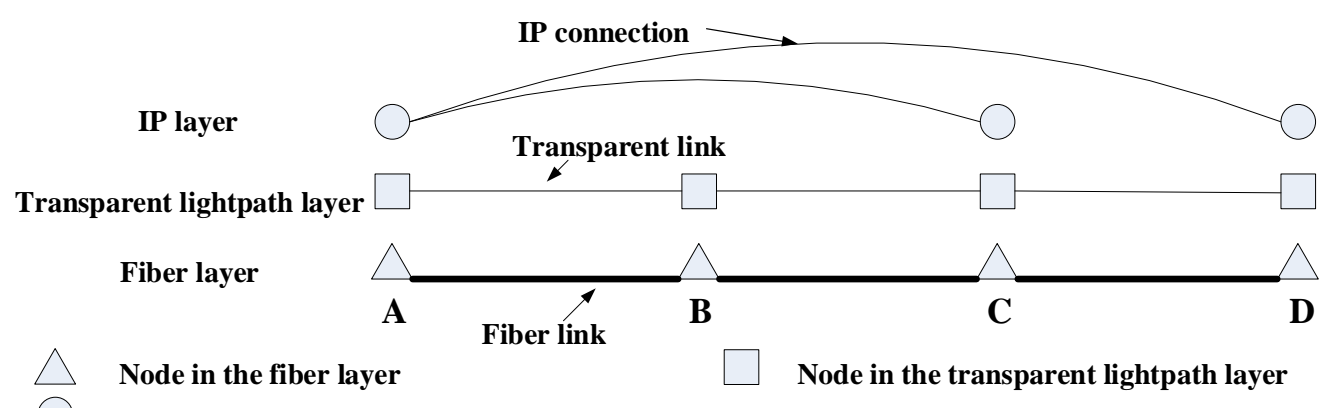

Node in the IP layer

(b)

Fig. 1. The layers of (a) the Tp-IPoWDM network (b) layers in the Tp-IPoWDM network

In the Tl-IPoWDM network as shown in Fig. 2(b), four layers are considered, i.e. the fiber layer, the TPL layer, the translucent lightpath (TLL) layer and the IP layer. The definition of the fiber layer is the same as that in the Tp-IPoWDM network. In the TPL layer, a virtual link still corresponds to a transparent lightpath but the end nodes of transparent lightpaths cannot only be the transponders but also the $3 \mathrm{R}$ regenerators. For example, the end nodes of virtual link (AB) in the TPL layer are a transponder and a 3R regenerator. In the TLL layer, a virtual link can be a translucent lightpath or a transparent lightpath. A translucent lightpath can be defined as a sequence of two or more transparent lightpaths that undergo 3R regeneration. Ends of a virtual link in the TLL layer are only transponders. In the IP layer, IP connection is established between IP nodes (core routers) based on the topology given by the TLL layer.

In the Tp-IPoWDM network, we only consider the energy consumed in the intermediate nodes. This consists of the power consumption of IP routers (for routing, grooming or signal regeneration), the transponders (for OEO signal conversion and signal transmission or 
reception) and the optical switches, denoted by $P_{\mathrm{I}}, P_{\mathrm{T}}$ and $P_{\mathrm{O}}$ respectively. In the Tl-IPoWDM network, the power consumptions of the $3 \mathrm{R}$ regenerators also need to be additionally considered (apart from the three parameters mentioned for the Tp-IPoWDM network). This is denoted by $P_{\mathrm{R}}$.

The assumptions of power consumption with constant values are made in the following. As the EDFAs are already deployed in the network, their power consumption is constant. It is noted that we only consider two fibers in each link in our study. Each fiber transmits in a different direction. Therefore, switching off EDFAs is not considered in cases when fibers become idle. Under any traffic grooming methods, the power consumption for the traffic processing at end nodes is constant. Therefore, we do not consider the power consumption of the EDFAs and traffic processing at end nodes.

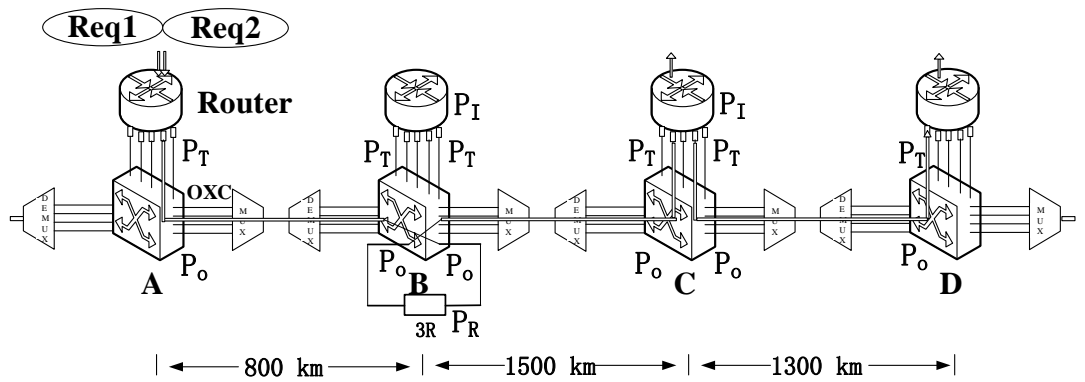

(a)

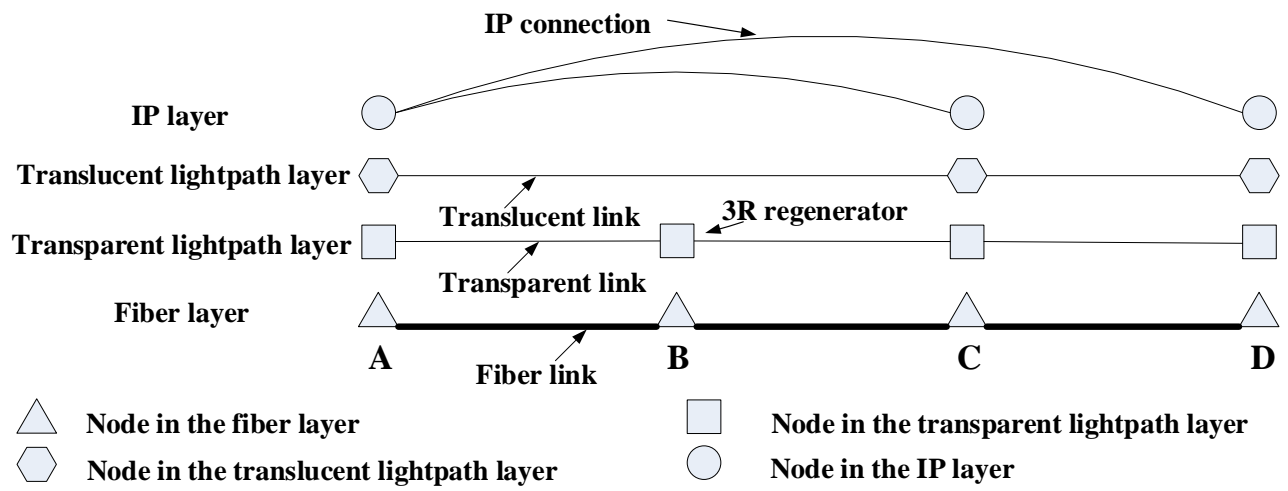

(b)

Fig. 2. The models of (a) the Tl-IPoWDM network (b) layers in the Tl-IPoWDM network

In Fig. 1(a), there are two IP connection requests. IP connection request 1 is from $\mathrm{A}$ to $\mathrm{C}$ with bandwidth requirement $r_{1}$; IP connection request 2 is from $\mathrm{A}$ to $\mathrm{D}$ with bandwidth requirement $r_{2}$. The total bandwidth of connection request 1 and 2 is smaller than that of a wavelength channel. It is assumed that the transparent lightpath length restriction is $2000 \mathrm{~km}$ in the TPL layer [22]. In the Tp-IPoWDM network, three lightpaths are established to provide bandwidth for the IP layer - one from A to B, one from B to C and one from $\mathrm{C}$ to $\mathrm{D}$, where two transponders and two optical switches are required for each transparent lightpath. IP connection request 1 travels through two transparent lightpaths, and is electronically switched at node B. IP connection request 2 travels through three transparent lightpaths, and is 
electronically switched at node $\mathrm{B}$ and $\mathrm{C}$. Therefore, the total power consumption is $6 P_{T}+6 P_{O}+r_{1} \cdot P_{I}+2 r_{2} \cdot P_{I}$.

In Fig.2 (a), the Tl-IPoWDM network has the same connection requests as the network in Fig. 1(a). In Fig. 2(a), the TLL layer can use 3R regenerator to extend the reach of the virtual link. There are two virtual links in the TLL layer. Virtual link AC consists of translucent lightpath $\mathrm{AC}$ which is composed of transparent lightpath $\mathrm{AB}$ and $\mathrm{BC}$. Virtual link $\mathrm{CD}$ consists of transparent lightpath $\mathrm{CD}$. The network requires 4 transponders, 6 optical switches and one $3 \mathrm{R}$ regenerator. Both connection requests do not need to be electronically switched at node $\mathrm{B}$. The total power consumption is $4 P_{T}+6 P_{O}+r_{2} \cdot P_{I}+P_{R}$.

\section{Mathematical ILP Formulation for the Tp-IPoWDM Network}

Minimizing power consumption in a bandwidth limited IP over WDM network is a bi-objective optimization problem, where the two objectives, maximizing the throughput and minimizing the power consumption, are conflicting in nature. In a transmission network, maximizing the throughput would be typically the main objective. Therefore, we use the lexicographic approach [19] in ILP. The network throughput is first optimized where the maximum throughput obtained is denoted by $\mathrm{T}$. The power consumption of the network is optimized under the constraint of Equation (1),

Subject to:

$$
f_{1}=T
$$

where $f_{1}$ denotes the sum of the bandwidth of all accepted IP connection requests.

In our study, two different ILP formulations are compared to optimize a Tp-IPoWDM network. If the paths have been calculated in advance, it can be denoted by a variable. This is named as the path based ILP (PB-ILP) method. If the paths are not given in advance, ILP needs to calculate paths for a given graph in the optimization process. This is named as the link based ILP (LB-ILP) method.

Since the paths have been calculated in advance, the complexity of PB-ILP is lower than that of LB-ILP. However, PB-ILP may only provide a sub-optimal solution if a limited number of paths is considered [21]. In the real IP over WDM network, the transparent lightpath length is constrained by the physical impairment. In this work, we will compare PB-ILP and LB-ILP in the green Tp-IPoWDM network.

In the following, LB-ILP and PB-ILP formulations for the Tp-IPoWDM network are given. We use the following indices.

$m$ and $n$ represent the ends of a fiber link;

$p$ and $q$ represent the ends of a virtual link in the TLL layer;

$i$ and $j$ represent the ends of a virtual link in the TPL layer;

$s$ and $d$ represent the ends of an IP connection request.

For the PB-ILP method, a set of $K$ shortest paths [23] are generated in advance for each node pair in a given physical topology. All transparent lightpaths are constrained to follow one of these paths. 


\section{Given Parameters:}

$G(V, E)$ physical topology, where $V$ is the set of network nodes and $E$ is the set of directed physical links connecting the nodes.

$N$ the number of wavelengths per fiber.

$B$ capacity of each wavelength channel.

$\varphi \quad$ the maximum transparent reach in the optical network.

$x \quad$ the basic bandwidth granularity of an IP connection.

$\wedge$ traffic demand matrix. $\wedge_{s d}$ is the number of $x$ Gbps connection requests from node $s$ to $d$. In this work, $x$ is set as 2 .

$r_{s d}^{t} \quad t^{t h}\left(t \in\left[1, \wedge_{s d}\right]\right)$ IP connection request from node $s$ to $d$.

$F_{m n}$ if there is a fiber from node $m$ to $n, F_{m n}=1$; otherwise, $F_{m n}=0$.

$F_{m n}^{w}$ if there is wavelength $w$ on fiber $(m, n), F_{m n}^{w}=1$; otherwise, $F_{m n}^{w}=0$.

$D_{m n}$ the length of the fiber between node $m$ and $n$.

$P_{i j, k}$ the $k^{\text {th }}(k=1, \ldots, K)$ shortest path in the fiber layer from node $i$ to $j$

$D_{i j, k}$ the length of $P_{i j, k}$.

$X_{i j, k}^{l}$ if the $k^{\text {th }}$ path from node $i$ to $j$ uses link $l$ in the fiber layer, $X_{i j, k}^{l}=1$; otherwise, $X_{i j, k}^{l}=0$.

$T$ network throughput.

\section{Decision variables:}

$\lambda_{s d}^{t}$ if the $t^{t h}\left(t \in\left[1, \wedge_{s d}\right]\right)$ IP connection request from node $s$ to $d$ is accepted, $\lambda_{s d}^{t}=1$; otherwise, $\lambda_{s d}^{t}=0$.

$U_{i j}^{s d, t}$ if $r_{s d}^{t}$ traverses through a lightpath from node $i$ to $j, U_{i j}^{s d, t}=1$; otherwise, $U_{i j}^{s d, t}=0$.

$L_{i j}^{w} \quad$ the number of lightpaths from node $i$ to $j$ using wavelength $w\left(L_{i j}^{w} \in\{0,1\}\right.$ for LB-ILP).

$F_{m n}^{i j, w}$ if a lightpath from node $i$ to $j$ traverses fiber link $(m, n)$ using wavelength $w, F_{m n}^{i j, w}=1$; otherwise, $F_{m n}^{i j, w}=0$.

$O_{i j, k}^{w}$ if a lightpath uses wavelength $w$ and follows path $P_{i j, k}, O_{i j, k}^{w}=1$; otherwise, $O_{i j, k}^{w}=0$.

$f_{1} \quad$ the sum of bandwidth of all accepted IP connection requests.

$f_{2}$ the network power consumption of the Tp-IPoWDM network calculated by the LB-ILP.

$f_{3}$ the network power consumption of the Tp-IPoWDM network calculated by the PB-ILP.

$f_{4}$ the network power consumption of the Tl-IPoWDM network calculated by the PB-ILP.

\section{The lexicographic approach}

There are two objectives in energy-efficient traffic grooming in bandwidth constraint IP over WDM networks. One is to maximize the network throughput, which is presented in (2); 
maximize:

$$
f_{1}=\sum_{t, s, d} x \cdot \lambda_{s d}^{t}
$$

The other is to minimize the network power consumption. In this work, we adopt the method in [8] to calculate the power consumption of the Tp-IPoWDM network. For the LB-ILP, it is presented in (3).

minimize:

$$
f_{2}=2 P_{T} \sum_{i, j} \sum_{w} L_{i j}^{w}+P_{O} \sum_{i, j} \sum_{w}\left[L_{i j}^{w}+\sum_{m, n} F_{m n}^{i j, w}\right]+P_{I}\left[\sum_{s, d, t} \sum_{i, j} x \cdot U_{i j}^{s d, t}-T\right]
$$

In Equation (3), the first term is the total power consumption of transponders in the network; the second one is the power consumption of optical switches; the third one evaluates the power consumption of electronically switched traffic (EST) in the intermediate nodes.

For PB-ILP, the Tp-IPoWDM network power consumption is presented in (4).

minimize:

$$
f_{3}=2 P_{T} \cdot \sum_{i, j} \sum_{w} \sum_{k} O_{i j, k}^{w}+P_{O} \sum_{i, j} \sum_{k} \sum_{w} O_{i j, k}^{w}\left[1+\sum_{l} X_{i j, k}^{l}\right]+P_{I}\left[\sum_{s, d, t} \sum_{i, j} x \cdot U_{i j}^{s d, t}-T\right]
$$

In Equation (4), the network power consumption is composed of three parts. The first term is the power consumption of transponders; the second term is the power consumption of optical switches; the third term is the power consumption of electronically switched traffic (EST) in the intermediate nodes.

Under the constraint of Eq. (1), the lower bound of the network power consumption can be optimized using Eq. (3) and Eq. (4) under LB-ILP and PB-ILP respectively. The resulting lower bound of the network power consumption with the maximum throughput is denoted by $\Phi$ watts.

If the network capacity is enough, Equation (1) will force all the connection demands to be accepted. The lexicographic approach will be equivalent to the single objective optimization method in which the network energy is minimized with the Constraint Function (5) [2] [8].

Subject to:

$$
\lambda_{s d}^{t}=1 \quad \forall t, s, d
$$

\section{LB-ILP formulations for the Tp-IPoWDM network}

\section{Constraints :}

-- Routing transparent lightpaths in the fiber layer 


$$
\begin{aligned}
& \sum_{n \in V: m \neq n} F_{m n}^{i j, w}-\sum_{n \in V: m \neq n} F_{n m}^{i j, w}=\left\{\begin{array}{cc}
L_{i j}^{w} & m=i \\
-L_{i j}^{w} & m=j \\
0 & \text { otherwise }
\end{array} \quad \forall i, j, w: i \neq j\right. \\
& \sum_{i \in V} \sum_{j \in V: i \neq j} F_{m n}^{i j, w} \leq F_{m n}^{w} \quad \forall m, n, w: m \neq n \\
& \sum_{m \in V} \sum_{n \in V: m \neq n} F_{m n}^{i j, w} \cdot D_{m n} \leq \varphi \quad \forall i, j, w: i \neq j
\end{aligned}
$$

Constraint (6) ensures the flow conservation in the TPL layer. The wavelength conflict constraint is given in Equation (7). In Constraint (8), the maximum length of a transparent lightpath is $\varphi$.

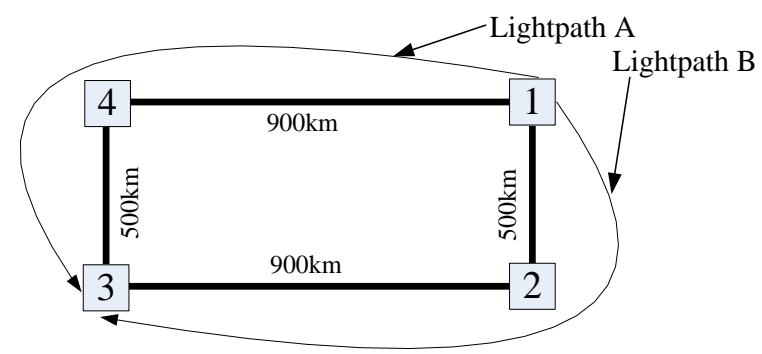

Fig. 3. Description of number restriction of transparent lightpaths in LB-ILP method

It is noted that the value of $L_{i j}^{w}$ must be 1 or 0 in Eq. (6) for LB-ILP. For example, a four-node WDM network with four fiber links is shown in Fig. 3. Two lightpaths, e.g. transparent lightpath A and transparent lightpath B, can be set up from node 1 to 3 . Both transparent lightpaths can use wavelength $\mathrm{w}$ without violating the wavelength conflict constraint. For $i=1, j=3$, and $w=1, \sum_{m \in V} \sum_{n \in V: m \neq n} F_{m n}^{i j, w} \cdot D_{m n}=2800 \mathrm{~km}$. If $\varphi=2000 \mathrm{~km}$, Equation (8) is not satisfied. Under this constraint, both transparent lightpaths will not be set up. However, both transparent lightpaths have the equal length of $1400 \mathrm{~km}$. The length restriction is satisfied. To avoid all transparent lightpaths being blocked from node 1 to 3 , the value of $L_{i j}^{w}$ must be 0 or 1 . Therefore, with the same wavelength, only one lightpath can be set up for connecting two nodes. Otherwise, the length restriction of Equation (8) forces some node pairs to stay unconnected even if all transparent lightpath lengths satisfy the length restriction. This will affect the path selectivity in the fiber layer.

-- Routing IP connection requests in the TPL layer

$$
\sum_{j \in V: i \neq j} U_{i j}^{s d, t}-\sum_{j \in V: i \neq j} U_{j i}^{s d, t}=\left\{\begin{array}{cc}
\lambda_{s d}^{t} & i=s \\
-\lambda_{s d}^{t} & i=d \\
0 & \text { otherwise }
\end{array} \quad \forall s, d: s \neq d \quad \forall t \in\left[1, \wedge_{s d}\right]\right.
$$




$$
\sum_{t} \sum_{s, d} x \times U_{i j}^{s d, t} \leq \sum_{w} L_{i j}^{w} \times B \quad \forall i, j
$$

Constraint (9) is responsible for routing small granularity IP connection requests in the TPL layer. Constraint (10) ensures that the accepted traffic cannot exceed the overall capacity of the established transparent lightpaths.

\section{PB-ILP formulations for the Tp-IPoWDM network}

\section{Constraints :}

-- Routing transparent lightpaths in the fiber layer

$$
\begin{array}{cr}
\sum_{i j} \sum_{k} O_{i j, k}^{w} \cdot X_{i j, k}^{l} \leq 1 & \forall l, w \\
O_{i j, k}^{w} \cdot D_{i j, k} \leq \varphi & \forall i, k, w, j \\
L_{i j}^{w}=\sum_{k} O_{i j, k}^{w} &
\end{array}
$$

Constraint (11) gives the wavelength conflict constraint. Constraint (12) restricts the length of a transparent lightpath. Equation (13) describes the relationship between $L_{i j}^{w}$ and $\sum_{k} O_{i j, k}^{w}$.

Compared with LB-ILP, the value of $L_{i j}^{w}$ can be greater than 1 in PB-ILP.

-- Routing IP connection requests in the TPL layer

We use Constraint (9) and (10) in the LB-ILP formulation.

\section{Numerical Results for the Tp-IPoWDM Network}

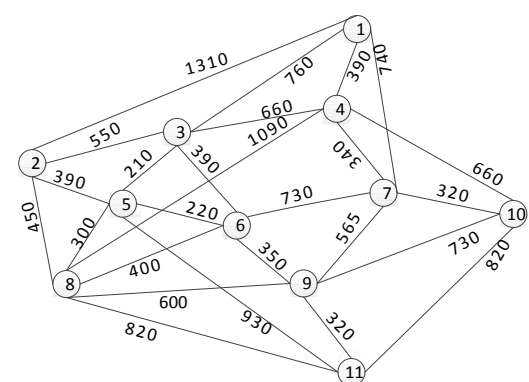

(a) $\operatorname{CosT} 239$

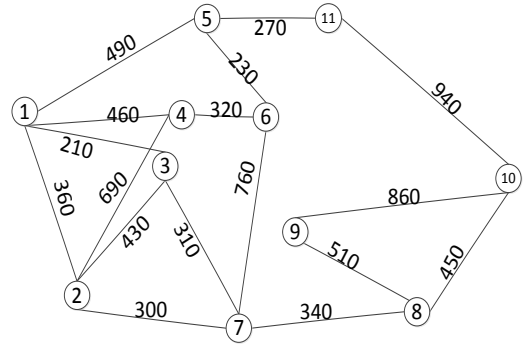

(b)11-node random network

Fig. 4. The topology of two networks $(\mathrm{km})$ 
To solve the ILP problems above, the commercial ILP solver, "CPLEX", is used on a computer equipped with two Xeon CPUs and 48GB RAM. In this work, we report on the results obtained using two network topologies, (i) the 11-node COST239 network of Fig. 4(a) with 26 links with average nodal degree of 4.73 and (ii) the 11-node random network of Fig. 4(b) with 17 links with average nodal degree of 3.09. The length of each link has been shown in the respective figures. It is assumed that every link has one fiber in each direction and each fiber has $N$ wavelengths. The bandwidth of each wavelength channel is $10 \mathrm{Gbps}$. For every node pair, 10 shortest paths are calculated in advance [23]. The reach of the optical signal is assumed to be limited to $2000 \mathrm{~km}$ [22]. The power consumption value of IP routers is assumed to be $14.5 \mathrm{~W}$ per Gbps. Each WDM transponder is $34.5 \mathrm{~W}$, and each optical switch is $1.5 \mathrm{~W}$ per wavelength [8].

In Table 1, the traffic demand matrix (as in [24]) for the COST239 network topology is given. Here, only one bandwidth granularity of $2 \mathrm{Gbit} / \mathrm{s}$ is considered in the IP core network. If a higher speed IP connection is required, it can be configured as a combination of multiple IP connections with the basic bandwidth granularity of $2 \mathrm{Gbps}$. The total amount of traffic is 2000Gbps. For the random network of Fig. 4(b), we use a randomly generated traffic demand matrix as shown in Table 2 . The total amount of traffic is $600 \mathrm{Gbps}$.

Table 1. Traffic Matrix for COST239 (granularity of 2 Gbps)

\begin{tabular}{|l|l|l|l|l|l|l|l|l|l|l|l|}
\hline Node & 1 & 2 & 3 & 4 & 5 & 6 & 7 & 8 & 9 & 10 & 11 \\
\hline 1 & 0 & 1 & 1 & 3 & 1 & 1 & 1 & 35 & 1 & 1 & 1 \\
\hline 2 & 1 & 0 & 5 & 14 & 40 & 1 & 1 & 10 & 3 & 2 & 3 \\
\hline 3 & 1 & 5 & 0 & 16 & 24 & 1 & 1 & 5 & 3 & 1 & 2 \\
\hline 4 & 3 & 14 & 16 & 0 & 6 & 2 & 2 & 21 & 81 & 9 & 9 \\
\hline 5 & 1 & 40 & 24 & 6 & 0 & 1 & 11 & 6 & 11 & 1 & 2 \\
\hline 6 & 1 & 1 & 1 & 2 & 1 & 0 & 1 & 1 & 1 & 1 & 1 \\
\hline 7 & 1 & 1 & 1 & 2 & 11 & 1 & 0 & 1 & 1 & 1 & 1 \\
\hline 8 & 35 & 10 & 5 & 21 & 6 & 1 & 1 & 0 & 6 & 2 & 5 \\
\hline 9 & 1 & 3 & 3 & 81 & 11 & 1 & 1 & 6 & 0 & 51 & 6 \\
\hline 10 & 1 & 2 & 1 & 9 & 1 & 1 & 1 & 2 & 51 & 0 & 81 \\
\hline 11 & 1 & 3 & 2 & 9 & 2 & 1 & 1 & 5 & 6 & 81 & 0 \\
\hline
\end{tabular}

Table 2. Traffic Matrix for 11 node random network (granularity of 2 Gbps)

\begin{tabular}{|l|l|l|l|l|l|l|l|l|l|l|l|}
\hline Node & 1 & 2 & 3 & 4 & 5 & 6 & 7 & 8 & 9 & 10 & 11 \\
\hline 1 & 0 & 5 & 6 & 1 & 3 & 2 & 1 & 9 & 3 & 4 & 5 \\
\hline 2 & 3 & 0 & 2 & 1 & 4 & 5 & 1 & 2 & 2 & 3 & 3 \\
\hline 3 & 2 & 2 & 0 & 4 & 1 & 5 & 2 & 1 & 3 & 4 & 5 \\
\hline 4 & 1 & 0 & 1 & 0 & 2 & 2 & 5 & 3 & 5 & 5 & 2 \\
\hline 5 & 1 & 0 & 6 & 2 & 0 & 3 & 2 & 4 & 1 & 6 & 2 \\
\hline 6 & 1 & 3 & 1 & 2 & 3 & 0 & 3 & 6 & 4 & 1 & 5 \\
\hline 7 & 4 & 3 & 5 & 2 & 3 & 1 & 0 & 3 & 3 & 3 & 3 \\
\hline 8 & 2 & 2 & 0 & 0 & 1 & 3 & 4 & 0 & 4 & 3 & 2 \\
\hline 9 & 5 & 1 & 3 & 3 & 4 & 6 & 0 & 3 & 0 & 1 & 5 \\
\hline 10 & 3 & 3 & 2 & 2 & 3 & 4 & 2 & 1 & 2 & 0 & 4 \\
\hline 11 & 2 & 1 & 1 & 2 & 3 & 1 & 2 & 0 & 2 & 2 & 0 \\
\hline
\end{tabular}




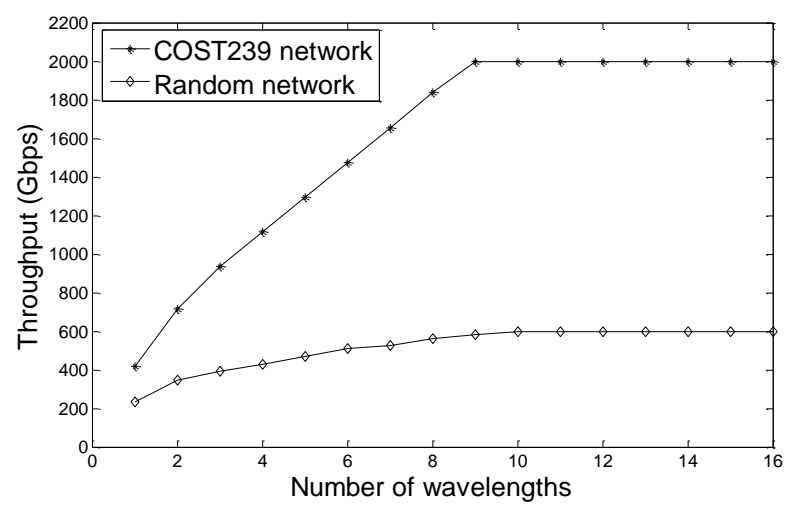

Fig. 5. Network throughput vs. number of wavelengths per fiber

Based on the parameter choices given above, we first optimize the network throughput for the two networks. The results obtained are shown in Fig. 5, indicating that though the (maximum) network throughput increases with the increasing number of wavelengths per fiber, there is a trend towards saturation. For example, the COST239 network can sustain a maximum throughput of about $2000 \mathrm{Gbps}$ as long as there are $\mathrm{N} \geq 9$ wavelengths per fiber. Similarly, the 11-node random network can accommodate all traffic load as long as there are $\mathrm{N} \geq 10$ wavelengths per fiber.

Subject to the calculated throughput value, the lower bounds on the network power consumption are then calculated. The network power consumption calculated under the lexicographic approach is denoted by $E_{\mathrm{b}}$. For comparison, $E_{\mathrm{s}}$ is used to denote the power consumption value of the network in which only the throughput is optimized. The values of $E_{s} / E_{b}$ under different ILP methods for two networks are plotted in Fig. 6(a) and Fig. 6(b). When $N=1$ in Fig. 6(a), the values of $E_{s} / E_{b}$ under two ILP methods are greater than 1.05. For other $\mathrm{N}$ values, the values of $\mathrm{Es} / \mathrm{Eb}$ are significantly more than 1 under both ILP methods in both figures. We can conclude that there are multiple path combinations satisfying the maximizing network throughput objective. With the lexicographic approach, the network can select the combination with the minimum power consumption. As Es is calculated based on a randomly selected result out of the multiple combinations which maximize the throughput, we do not discuss the trends of simulation results further.

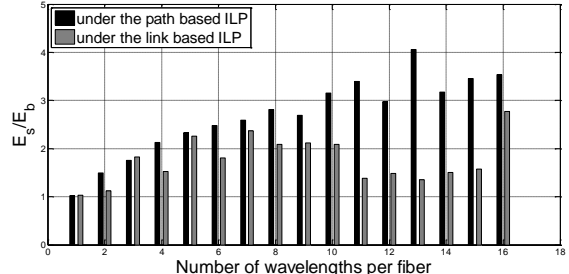

(a) COST239 network

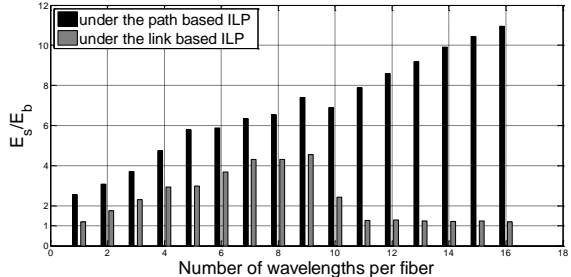

(b) Random network

Fig. 6. The value of $E_{\mathrm{s}} / E_{\mathrm{b}}$ 
It would also be interesting to compare the performance of LB-ILP and PB-ILP for energy minimization in bandwidth constrained IP over WDM networks. This has been done in Fig. 7(a) and Fig. 7(b) which compare the network power consumption $E_{b}$ for both network topologies. In both networks, performance trends are similar for both ILPs. In the same network, $E_{\mathrm{b}}$ under both ILP methods starts with the same value when $N=1$ and increases as $N$ increases. The values of $E_{\mathrm{b}}$ reach their maximum when the network capacity is just enough for the traffic load (for the COST239 network, $N=9$; for the random network, $N=10$ ). Fig. 7(a) shows the LB-ILP method always consumes more energy than the PB-ILP method in the COST239 network. When $N=9$, the $E_{\mathrm{b}}$ value difference is the highest. As the value of $N$ continues increasing, values of $E_{\mathrm{b}}$ under both methods start to decrease and ultimately reach the saturation values. When $\mathrm{N}=16$, the power consumption value under the PB-ILP method is still significantly less than that under the LB-ILP method. However, for the random network in Fig. 7(b), the performance improvement is marginal for PB-ILP over LB-ILP.

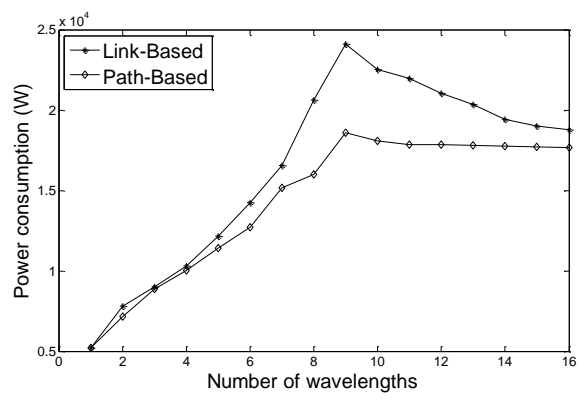

(a) COST239 network

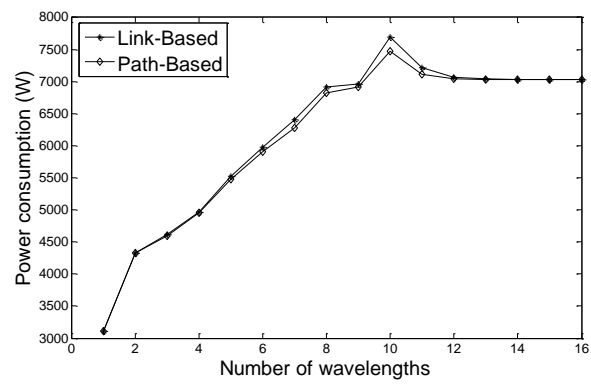

(b) Random network

Fig. 7. Network power consumption vs. number of wavelengths

As power consumption values of transponders and routers are several times greater than those of optical switches in network energy calculating Equation (3) and (4), we plot the number of transparent light paths vs. number of wavelengths per fiber in Fig. 8 and the average number of electrical hops (ANEH) vs. number of wavelengths per fiber in Fig. 9 for further studying the reason of variation in the network power consumption values in Fig. 7. In

Fig. 9, The ANEH is denoted by $\bar{H}$ in (15).

$$
\bar{H}=\sum_{s, d, t} \sum_{i, j} x \cdot U_{i j}^{s d, t} / T
$$

This reflects the level of multi-electrical-hop routing utilized in the IP layer.

In the Tp-IPoWDM network, a transparent lightpath uses two transponders. The proportion between numbers of lightpaths under two ILP methods will result in the same proportion between transponder power consumptions. Fig. 8(a) and Fig. 8(b) have the similar trends as Fig. 7(a) and Fig. 7(b) respectively. The only difference is that the performance difference between two methods in Fig. 8(a) is not as large as that in Fig. 7(a) when $N=9$. There may be other reasons for the large power consumption difference between two ILP methods. 


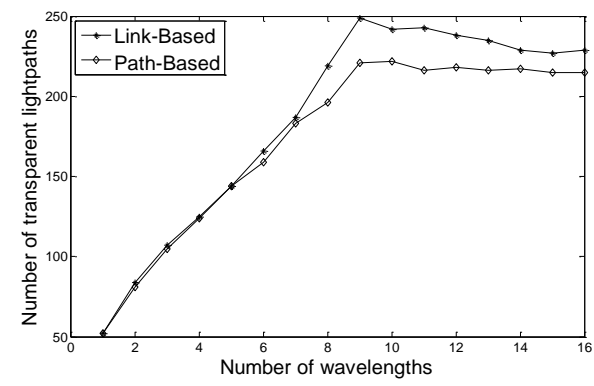

(a) COST239 network

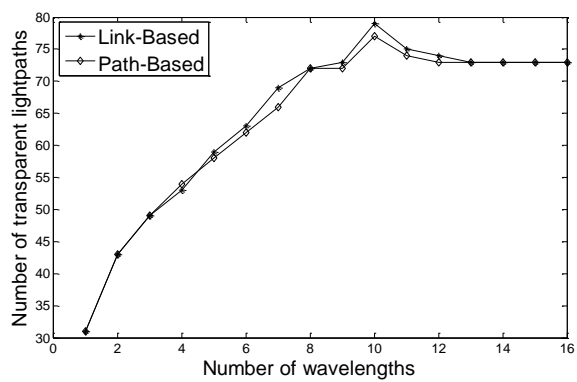

(b) Random network

Fig. 8. The number of transparent lightpaths vs. number of wavelengths

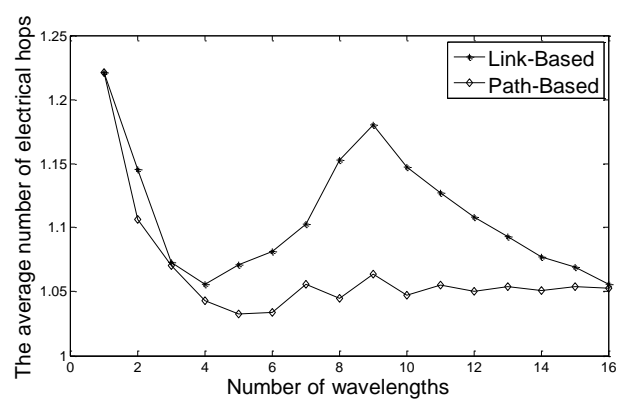

(a) COST239 network

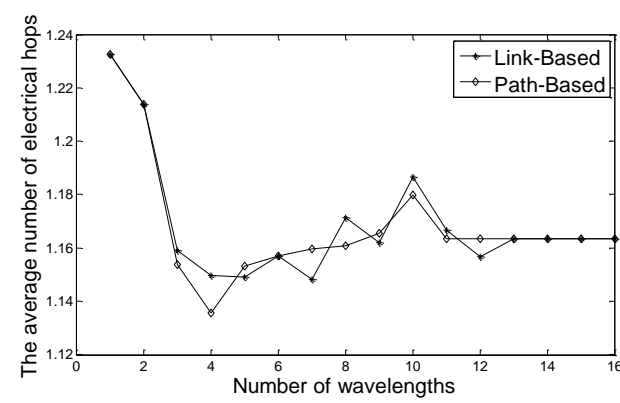

(b) Random network

Fig. 9. The average number of electrical hops vs. number of wavelengths

In Fig. 9(a), for the COST239 network, both methods have the same biggest value of ANEH when $N=1$. The reason is that the network has only one wavelength on each fiber and tends to set up one hop lightpaths for maximizing the network throughput. Most of the traffic can only be switched by routers if these are required to travel two or more fiber links. As the number of wavelengths increases, more multi-hop lightpaths can be set up without sacrificing the network throughput; the value of ANEH then starts to decrease. It is interesting that the value of ANEH starts to increase when $N$ is greater than 4 for the LB-ILP method and when $N$ is greater than 6 for the PB-ILP method. This is because the first objective is to maximize the network throughput where we have assumed that the network throughput is the sum of the bandwidth of the accepted IP connections regardless of other aspects of IP paths. After analyzing the rejected connection requests, we find two kinds of connections that are more likely to be rejected. The first kind of connections has few path selections in the physical topology. For example, we calculate 10 paths for each node pairs in advance. However, there may be only 5 paths satisfying the length restriction between node 9 and 10 in Fig. 4(a). The fewer path selections a connection has, the higher the probability will be that a connection may be rejected. The second kind of connections will connect heavy load node pairs. In this case, there are many connections requests between the heavy load end node pairs and it is possible for a new connection request to be blocked. For example, in Table 1, there are 812-Gbps connection request between 11 and 10 . However from node 2 to 1 , there is only 1 connection 
request. We name both kinds of connections as difficult connection requests, while the other connections are named as easy connection requests. After all the easy connection requests are accepted, the network has to accept the more difficult connection requests in order to increase the network throughput as the value of $N$ increases. Routing difficult connections will encounter more congestion situations. As the fiber layer has fewer path selections than the TPL layer, the network will use routers to switch more difficult connection requests to improve the network throughput when the number of wavelengths per fiber increases.

It is also shown that the value of ANEH under LB-ILP increases fast after $N=4$. For the network under the PB-ILP method, the value of ANEH decreases until $N=6$. After that, the value of ANEH increases slightly and fluctuates around 1.05. On the other hand, the value of ANEH under the LB-ILP method reaches 1.18 when $N=9$. At this time, values of ANEH between two ILP methods have the biggest difference. The poorer performance of LB-ILP is due to the constraint of $L_{i j}^{w} \in\{0,1\}$ as discussed in Sec. III. This restricts the path selection, especially when there is not enough wavelength resource. Therefore, the IP over WDM network has to do more multi-electrical-hop switching in order to increase the network capacity.

When $N$ is greater than 9 , the network capacity is bigger than the traffic load and the wavelength resource is more than sufficient. For the LB-ILP method, increasing wavelength choices can offset the path limitation caused by the constraint of $L_{i j}^{w} \in\{0,1\}$. As a result, the value of ANEH under the LB-ILP starts to decrease. When $N=16$, both methods have similar values of ANEH. From Fig. 7(a), Fig. 8(a) and Fig. 9(a), we may conclude that when $N=16$, the power consumption difference between two methods is due to the different number of lightpaths (transponders) used, which means that the PB-ILP method can have better lightpath combinations and use fewer lightpaths than the LB-ILP method. It may be noted that the values of ANEH under both ILP methods are very close in Fig. 9(b).

Our simulation results show that the PB-ILP method significantly outperforms the LB-ILP method in the COST239 network. With the PB-ILP method, the network uses fewer lightpaths to achieve lower overall power consumption. On the contrary, both ILP methods show quite similar performances in the random network. This is because the random network has a smaller average nodal degree and less path choices than the COST239 network. Actually, in the random network, the PB-ILP method cannot use more than 2 paths for some node pairs, e.g., node pair $(5,11),(7,10)$, etc. Without enough path selection choices, the PB-ILP method cannot outperform the LB-ILP method.

\section{Mathematical (ILP) Formulation for the TI-IPoWDM Network}

Compared with the Tp-IPoWDM network, the Tl-IPoWDM network can use not only routers but also $3 \mathrm{R}$ regenerators for the optical signal regeneration. To describe multi-layer routing in the Tl-IPoWDM network, a four-layer model is given in Fig. 2(b). Compared with the three-layer model of the Tp-IPoWDM network, it has one more layer, e.g. the translucent lightpath (TLL) layer. The TLL layer is between the TPL layer and the IP layer in Fig. 2(b). In order to give the flow conservation relationship between virtual links in the TPL layer and links in the TLL layer, it is a must to denote a virtual link (transparent lightpath) in the TPL layer. However, a transparent lightpath cannot be denoted as a variable in the ILP formulation before it is calculated except that there is only one path between two nodes. Obviously, there are more than one paths between two nodes in the network. Therefore, PB-ILP formulations have to be 
used. In this work, the end nodes of a virtual link in the TLL layer are denoted by $p$ and $q$. Apart from those given in Sec. IV, the following decision variables are added.

\section{Decision variables:}

$U_{p q}^{s d, t}$ if $r_{s d}^{t}\left(t \in\left[1, \wedge_{s d}\right]\right)$ uses a virtual link in the TLL layer from node $p$ to $q, U_{p q}^{s d, t}=1$; otherwise, $U_{p q}^{s d, t}=0$ 。

$v_{p q} \quad$ the number of virtual links in the TLL layer from node $p$ to $q$.

$L_{i j, k}^{p q} \quad$ the number of virtual links in the TLL layer from $p$ to $q$ that use transparent lightpaths on path $P_{i j, k}$.

$L_{i j, k, w}^{p q}$ if a virtual link in the TLL layer from node $p$ to $q$ is served by a transparent lightpath from node $i$ to $j$ using wavelength $w$ and path $P_{i j, k}, L_{i j, k, w}^{p q}=1$; otherwise, $L_{i j, k, w}^{p q}=0$.

\section{Constraints:}

-- Routing transparent lightpaths in the fiber layer

Use the same constraints of Equation (11) and (12) in the PB-ILP formulations for the Tp-IPoWDM network.

-- Routing virtual links of the TLL layer based on the virtual topology given by the TPL layer

$$
\begin{array}{cc}
O_{i j, k}^{w}=\sum_{p q} L_{i j, k, w}^{p q} & \forall i, j, k, w \\
L_{i j, k}^{p q}=\sum_{w} L_{i j, k, w}^{p q} & \forall i, j, p, q, k \\
\sum_{k} \sum_{j \in V: i \neq j} L_{i j, k}^{p q}-\sum_{k} \sum_{j \in V: i \neq j} L_{j i, k}^{p q}=\left\{\begin{array}{cc}
v_{p q} & i=p \\
-v_{p q} & i=p \\
0 & \text { otherwise }
\end{array}\right. & \forall p, q
\end{array}
$$

Constraint (16) ensures that a transparent lightpath can only be used by one virtual link in the TLL layer. Equation (17) describes the relationship between $L_{i j, k, w}^{p q}$ and $L_{i j, k}^{p q}$. Constraint (18) ensures the flow conservation relationship between virtual links in the TLL layer and virtual links in the TPL layer.

Routing IP connection requests in the virtual topology provided by the TLL layer

$$
\sum_{q \in V: p \neq q} U_{p q}^{s d, t}-\sum_{q \in V: p \neq q} U_{q p}^{s d, t}=\left\{\begin{array}{cc}
\lambda_{s d}^{t} & p=s \\
-\lambda_{s d}^{t} & p=d \\
0 & \text { otherwise }
\end{array} \quad \forall s, d \quad \forall t \in\left[1, \vee_{s d}\right]\right.
$$




$$
\sum_{t} \sum_{s, d} U_{p q}^{s d, t} \times x \leq v_{p q} \times B \quad \forall p, q
$$

Constraint (19) ensures the flow conservation relationship between IP connections and virtual links in the TLL layer. Constraint (20) ensures that the aggregate traffic between two routers in the IP layer cannot exceed the capacity of the TLL layer virtual links which connect the same routers.

Objective:

minimize:

$$
f_{4}=2 P_{T} \cdot \sum_{p, q} v_{p q}+P_{O} \sum_{w} \sum_{i, j, k} O_{i j, k}^{w}\left[1+\sum_{l} X_{i j, k}^{l}\right]+P_{I}\left[\sum_{s, d, t, p, q} x \cdot U_{p q}^{s d, t}-T\right]+P_{R} \sum_{p, q}\left[\sum_{i, j, k} L_{i j, k}^{p q}-v_{p q}\right]
$$

The network power consumption in Equation (21) is composed of four parts. The first term is the power consumption of transponders; the second term is the power consumption of optical switches; the third term is the power consumption of routers in the intermediate nodes; the fourth term is the power consumption of $3 \mathrm{R}$ regenerators. The network power consumption is optimized under the constraint of Equation (1)

\section{Numerical Results for TI-IPoWDM Network}

In the Tl-IPoWDM network, the power consumption value of a regenerator is assumed to be $50 \mathrm{~W}$ per wavelength [8]. For studying the translucent network performance, we have run simulations based on the topology given in Fig. 4. However, it is shown that the power consumption of the Tl-IPoWDM network is nearly the same as that of the Tp-IPoWDM network. This is because path lengths of the COST239 network and the random network are short compared with the lightpath length limitation of $2000 \mathrm{~km}$. $3 \mathrm{R}$ regenerators are seldom used in both networks for saving energy. To show the advantage of the Tl-IPoWDM network, we choose to multiply the link lengths by 1.5 in both networks in Fig. 4.

Thanks to the switching capability of routers, the Tl-IPoWDM network and Tp-IPoWDM network with new link lengths have the same throughput as the Tp-IPoWDM network with original link lengths. Therefore, the network power consumption can be optimized according to capacity constraints in Fig. 5.

Fig. 10 shows the network power consumption vs. number of wavelengths per fiber for two networks under the Tp-IPoWDM network and Tl-IPoWDM network. Fig. 10(a) and Fig. 10(b) show similar characteristics. Firstly, all networks reach the highest power consumption values when the network capacity is just beyond the traffic load as shown in Fig. 5 (when $N=9$ in the COST239 network; when $N=10$ in the random network). Secondly, the Tl-IPoWDM network can significantly save a larger amount of power than the Tp-IPoWDM network. However, it is shown that, under the COST239 network topology, the power saving effect of the Tl-IPoWDM network decreases as the network capacity increases beyond the traffic load. To find out the reason, we study the variation of three evaluation metrics vs. number of wavelengths per fiber for the COST239 network in Fig. 11 and for the random network in Fig. 12. 


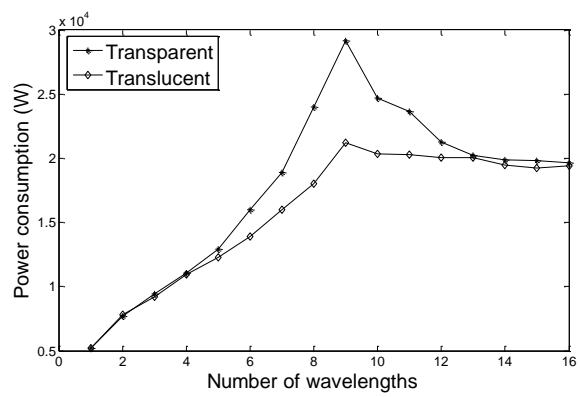

(a) COST239 network

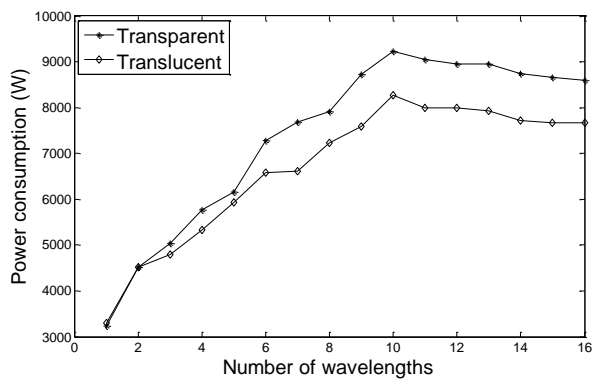

(b) Random network

Fig. 10. Network power consumption vs. number of wavelengths

As a virtual link in the TLL layer in the Tl-IPoWDM network can be a translucent lightpath or a transparent lightpath, we choose to compare the number of transponders used for the two networks in Fig. 11(a) and Fig. 12(a) rather than the number of lightpaths. Fig. 11(a) and Fig. 12(a) show similar trends as Fig. 10(a) and Fig. 10(b), respectively.

Fig. 11(b) and Fig. 12(b) show the relationship between the value of ANEH and the number of wavelengths per fiber for both network topologies. Under both network topologies, it is shown that the value of ANEH in the Tl-IPoWDM network is larger than that in the Tp-IPoWDM network. However, the way that the performance difference changes in the two networks is not the same. In Fig. 11(b), the maximum performance difference is reached when $N=9$. When the value of $N$ increases beyond 9, the difference between two ANEH values under the two networks decreases and the two ANEH values become only marginally different from each other. On the other hand, in Fig. 12(b), the value of ANEH in the Tp-IPoWDM network is about 0.2 higher than that in the Tl-IPoWDM network when $\mathrm{N}=16$. When the performance differences in Fig. 11(b) and Fig. 12(b) are large, it is observed that the number of used 3R regenerators is also large in Fig. 11(c) and Fig. 12(c). We may conclude that the Tl-IPoWDM network can manipulate 3R regenerators for setting up transparent lightpaths which will decrease the value of ANEH. As a result, router power is saved. In Fig. 10(b), Fig. 11(a) and Fig. 11(b), as the value of $N$ increases beyond 9, the Tp-IPoWDM network can improve the network energy efficiency with the decreasing number of transponders and decreasing value of ANEH. This is because the Tp-IPoWDM network can improve transparent lightpath combinations when the wavelength resource is sufficiently large. On the other hand, for the sparse random network topology in Fig. 10(b), the Tp-IPoWDM network cannot reach the network energy efficiency of the Tl-IPoWDM network even when $N=16$. At that point, the number of used $3 \mathrm{R}$ regenerators does not decrease significantly compared with the peak value of 12 in Fig. 12(c). This is because the sparse network has fewer path selections available than the dense network. Even with ample wavelength resources, it is still hard to reach some node pairs by using transparent lightpaths. In this case, the $3 \mathrm{R}$ regenerators are important for saving power in an IP over WDM network. 


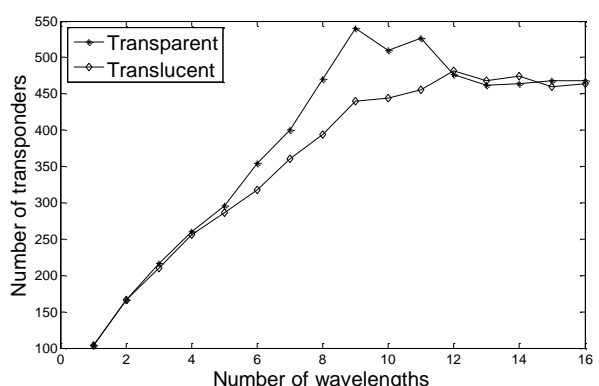

(a) The number of transponders vs. number of wavelengths

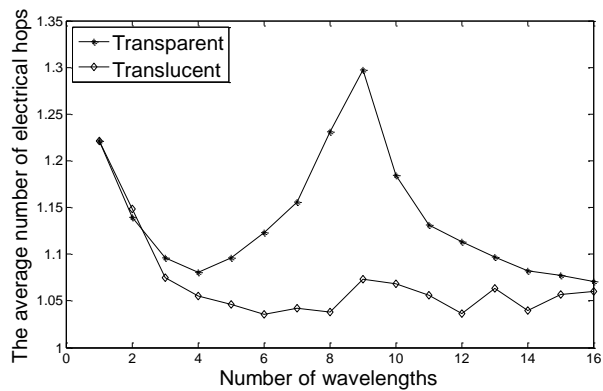

(b) The average number of electrical hops vs. number of wavelengths

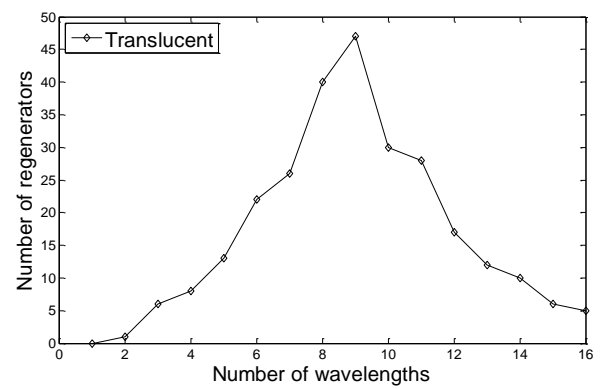

(c) The number of used $3 \mathrm{R}$ regenerators vs. number of wavelength

Fig. 11. Network performance of COST329 network

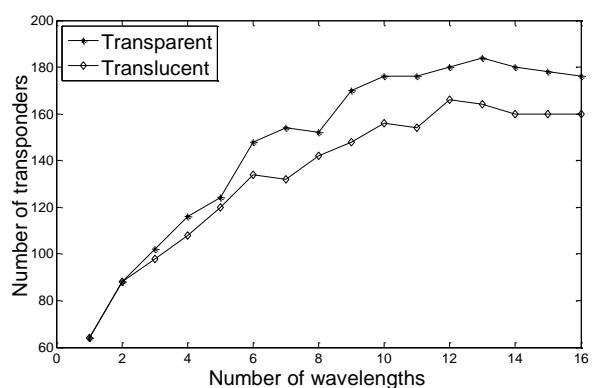

(a) The number of transponders vs. number of wavelengths

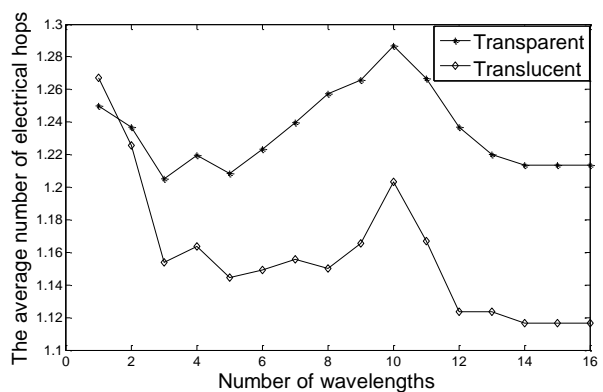

(b) The average number of electrical hops vs. number of wavelengths

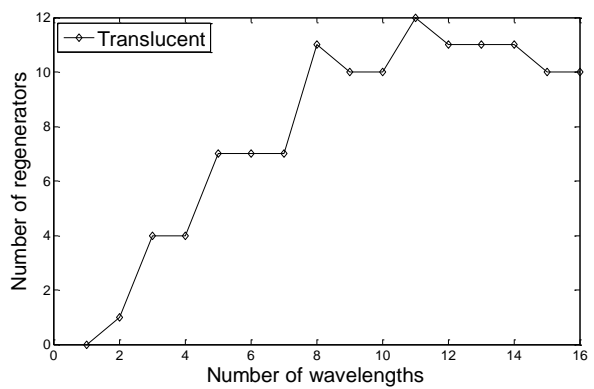

(c) The number of used $3 \mathrm{R}$ regenerators vs. number of wavelength

Fig. 12. Network performance of random network

\section{Conclusion}

For the energy optimization problem in bandwidth limited IP over WDM networks, the lexicographic approach is adopted to handle the ILP for the two opposing objectives, i.e. maximizing network throughput and minimizing network power consumption. The Tp-IPoWDM network and the Tl-IPoWDM network are investigated. For the Tp-IPoWDM networks, the LB-ILP and PB-ILP methods are compared. It is shown that the PB-ILP method can achieve higher energy efficiency than the link based ILP method in the dense network topology. This is because the PB-ILP method can have more path choices than the LB-ILP method under path length restriction in the dense network topology. However, for the sparse network topology which has limited path choices, the PB-ILP method cannot derive much advantage. For the Tl-IPoWDM network, only the PB-ILP method is used. The network performances are compared between the Tp-IPoWDM and Tl-IPoWDM networks. It is show 
that the Tl-IPoWDM network can outperform the Tp-IPoWDM network under both the dense network topology and the sparse network topology. In all networks, it is shown that the network energy efficiency can be improved by over-provisioning wavelength resources. Therefore, over-provisioning wavelengths can not only avoid congestion but also save energy. For the dense network topology, the network energy efficiency of the Tp-IPoWDM network can even be close to that of the Tl-IPoWDM network when the number of wavelengths per fiber is large enough. For the sparse IP over WDM network, the 3R regenerators become an important fact for saving power. It is shown that the Tl-IPoWDM network can save a significant amount of power compared with the Tp-IPoWDM network even when adequate provision is made for the wavelength resources. In future, we plan to extend our proposed approaches to elastic optical networks and consider the effect of 5G application[25-27] as well.

\section{References}

[1] R. S. Tucker, "Green optical communications-Part II: Energy limitations in networks," IEEE Journal of Selected Topics in Quantum Electronics, vol. 17, no. 2, pp. 261-274, 2011. Article (CrossRef Link)

[2] G. Shen, and R. S. Tucker, "Energy-minimized design for IP over WDM networks," IEEE/OSA Journal of Optical Communications and Networking, vol. 1, no. 1, pp. 176-186, 2009. Article (CrossRef Link)

[3] K. Y. Zhu, and B. Mukherjee, "Traffic grooming in an optical WDM mesh network," IEEE Journal on Selected Areas in Communications, vol. 20, no. 1, pp. 122-133, Jan, 2002. Article (CrossRef Link)

[4] B. Chen, S. K. Bose, W. D. Zhong, and H. Wang, "A new lightpath establishing method for dynamic traffic grooming under the overlay model," Photonic Network Communications, vol. 17, no. 1, pp. 11-20, 2009. Article (CrossRef Link)

[5] S. Huang, Deepa Seshadri, and Rudra Dutta. "Traffic grooming: a changing role in green optical networks." in Proc. of GLOBECOM'09, pp.1-6. Article (CrossRef Link)

[6] E. Yetginer, and G. N. Rouskas, "Power Efficient Traffic Grooming in Optical WDM Networks," in Proc. of IEEE GLOBECOM’09, pp. 1-6, 2009. Article (CrossRef Link)

[7] F. Farahmand, M. M. Hasan, I. Cerutti, J. P. Jue, and J. J. P. C. Rodrigues, "Differentiated Energy Savings in Optical Networks with Grooming Capabilities," in Proc. of IEEE GLOBECOM'10, pp. 1-5, 2010. Article (CrossRef Link)

[8] F. Musumeci, M. Tornatore, and A. Pattavina, "A power consumption analysis for IP-over-WDM core network architectures," IEEE/OSA Journal of Optical Communications and Networking, vol. 4, no. 2, pp. 108-117, 2012. Article (CrossRef Link)

[9] P. Chowdhury, M. Tornatore, A. Nag, E. Ip, T. Wang, and B. Mukherjee, "On the design of energy-efficient mixed-line-rate (MLR) optical networks," IEEE Journal of Lightwave Technology, vol. 30, no. 1, pp. 130-139, 2012. Article (CrossRef Link)

[10] Y. Wu, W. G. Hou, L. Guo, Y. J. Liu, and Z. M. Sun, "Green Grooming in Elastic Optical Networks," in Proc. of IEEE OFC'14, pp1-3, 2014. Article (CrossRef Link)

[11] F. Idzikowski, S. Orlowski, C. Raack, H. Woesner, and A. Wolisz, "Saving energy in IP-over-WDM networks by switching off line cards in low-demand scenarios," in Proc. of ONDM'10, pp. 1-6, 2010. Article (CrossRef Link)

[12] R. Bolla, R. Bruschi, A. Cianfrani, and M. Listanti, "Enabling backbone networks to sleep," IEEE Network, vol. 25, no. 2, pp. 26-31, 2011. Article (CrossRef Link)

[13] G. Rizzelli, A. Morea, M. Tornatore, and A. Pattavina, "Reach-Related Energy Consumption in IP-Over-WDM 100G Translucent Networks," IEEE/OSA Journal of Lightwave Technology, vol. 31, no. 11, pp. 1828-1834, 2013. Article (CrossRef Link) 
[14]P. Wiatr, P. Monti, and L. Wosinska, "Power savings versus network performance in dynamically provisioned WDM networks,” IEEE Communications Magazine, vol. 50, no. 5, pp. 48-55, 2012. Article (CrossRef Link)

[15] M. Xia, M. Tornatore, Y. Zhang, P. Chowdhury, C. U. Martel, and B. Mukherjee, "Green Provisioning for Optical WDM Networks," IEEE Journal of Selected Topics in Quantum Electronics, vol. 17, no. 2, pp. 437-445, 2011. Article (CrossRef Link)

[16]C. Cavdar, "Energy-efficient connection provisioning in WDM optical networks." in Proc. of $O F C^{\prime} 11$, pp. 1-3, 2011. Article (CrossRef Link)

[17] A. Fallahpour, H. Beyranvand, S. A. Nezamalhosseini, and J. A. Salehi, "Energy Efficient Routing and Spectrum Assignment With Regenerator Placement in Elastic Optical Networks," IEEE/OSA Journal of Lightwave Technology, vol. 32, no. 10, pp. 2019-2027, 2014. Article (CrossRef Link)

[18] M. M. Hasan, F. Farahmand, J. P. Jue, and J. J. P. C. Rodrigues, “A Study of Energy-Aware Traffic Grooming in Optical Networks: Static and Dynamic Cases,” IEEE Systems Journal, vol. 7, no. 1, pp. 161-173, 2013. Article (CrossRef Link)

[19] H. Isermann, "Linear lexicographic optimization," OR Spectrum, vol. 4, no. 4, pp. 223-228, 1982. Article (CrossRef Link)

[20] B. Chen, Z. Jiang, R. K. F. Teng, X. Lin, M. Dai, and H. Wang, “An energy efficiency optimization method in bandwidth constrained IP over WDM networks," in Proc. of IEEE ICICS'13, pp. 1-4, 2013. Article (CrossRef Link)

[21]R. M. Krishnaswamy, and K. N. Sivarajan, "Algorithms for routing and wavelength assignment based on solutions of LP-relaxations," IEEE Communications Letters, vol. 5, no. 10, pp. 435-437, Oct, 2001.Article (CrossRef Link)

[22] M. Yannuzzi, M. Quagliotti, G. Maier, E. Marín-Tordera, X. Masip-Bruin, S. Sánchez-López, J. Sole-Pareta, W. Erangoli, and G. Tamiri, "Performance of translucent optical networks under dynamic traffic and uncertain physical-layer information," in Proc. of ONDM'09, pp. 1-6, 2009. Article (CrossRef Link)

[23] J. Y. Yen, "Finding the k shortest loopless paths in a network," Management Science, vol. 17, no. 11, pp. 712-716, 1971. Article (CrossRef Link)

[24] A. K. Somani, "Survivability and Traffic Grooming in WDM Optical Networks," Cambridge, U. K.: Cambridge Univ. Press, 2006. Article (CrossRef Link)

[25] X. Ge, S. Tu, G. Mao, C. Wang and T. Han, "5G Ultra-Dense Cellular Networks," IEEE Wireless Communications, Vol. 23, No. 1, pp.72-79, Feb. 2016. Article (CrossRef Link)

[26] X. Ge, J. Yang, H. Gharavi and Y Sun, "Energy Efficiency Challenges of 5G Small Cell Networks,' IEEE communications Magazine, Vol. 55, No. 5, pp. 184-191, May 2017. Article (CrossRef Link)

[27] X. Ge, L Pan, Q. Li, G. Mao and Song Tu, "Multi-Path Cooperative Communications Networks for Augmented and Virtual Reality Transmission," IEEE Transactions on Multimedia, Vol 19, Issue 10, pp. 2345-2358, 2017. Article (CrossRef Link) 


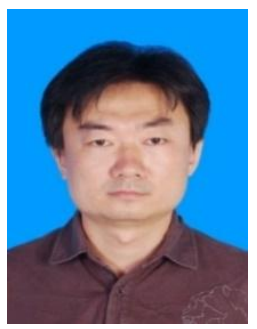

Bin Chen received the B.E. and M.S. degrees in electronic engineering in 1997 and 2002 from Lanzhou University. He got his Ph.D. degree in Communication Engineering from Nanyang Technological University in 2007. He is now an associate professor in Shenzhen University. His research interests include optical networking and cloud computing.

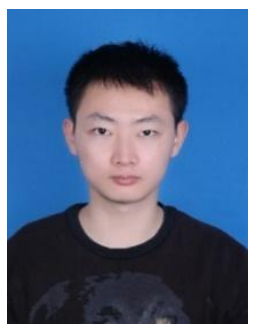

Zijian Yang received B.S. degree in Opto-Electronics Information Science and Engineering from Nanchang Hangkong University in 2016. His is now a Master student in Shenzhen University. His research interests include optical network optimization and cloud computing.

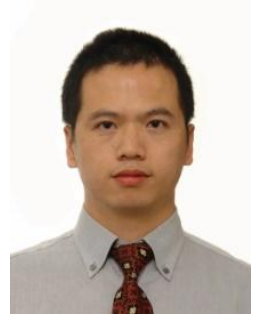

Rongping Lin received the Ph.D. degree from Nanyang Technological University, Singapore, in 2013. He is an Associate Professor with the School of Communication and information engineering, University of Electronic Science and Technology of China (UESTC), China. His research interests include traffic engineering and optimization design in communication networks.

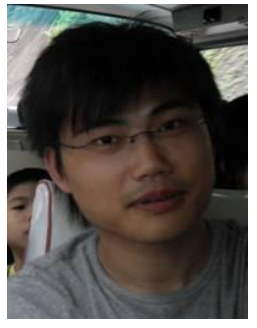

Mingjun Dai received the Ph.D degree in electronic engineering from City University of HongKong in 2012. He is now a lecturer with the College of Information Engineering, Shenzhen University, China. His research interests include cooperative relay network, network coding design, and optimization of wireless networks.

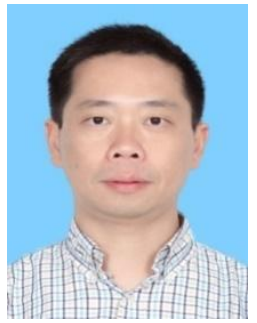

Xiao-Hui Lin received his B.S. and M.S. degrees in Electronics and Information Science from the Lanzhou University in 1997 and 2000, respectively. He got his Ph.D. in Electrical and Electronic Engineering from the University of HongKong in 2003. He is now an Associate Professor in the Faculty of Information Engineering, Shenzhen University in Guangdong, China. His research interests include mobile computing, wireless networking, and multimedia communication. In these fields, he has published more than 20 papers in international leading journals and refereed conferences. 


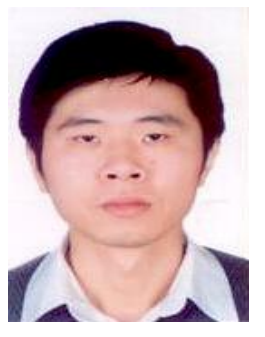

Gongchao Su received his B.S., M.S. and PhD degrees from the University of Electronic Science and Technology of China (UESTC), in 2000, 2003 and 2016, respectively, all in communications engineering. Since 2003 he has been with the Faculty of Information Engineering, Shenzhen University, where he is currently a lecturer. His research interests include modeling, performance analysis and optimization of wireless heterogeneous networks.

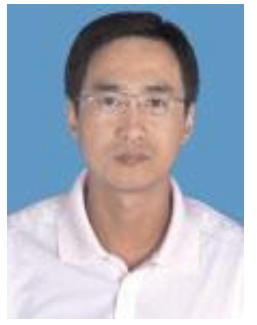

Hui Wang received his B.S., M.S., and Ph.D. from Xi'an Jiaotong University, in 1990, 1993, and 1996, respectively, all in Telecommunication. He is now a Professor and Dean of the Faculty of Information Engineering, Shenzhen University. His research interests include wireless communication, signal processing, and distributed computing systems, in which, he is author or co-author of more than 50 international leading journals, conferences and book chapters. 